\title{
Menopause and sleep disorders: does peri and post menopause influence the development of OSA and/ or insomnia?
}

\begin{abstract}
Introduction: The sleep quality decreases when women are in the peri or post menopause, possibly due to the decrease of the estrogen and progesterone levels that occur at this stage of their lives.

Aim: Evaluate if women after entering peri or post menopause, have a higher probability of having sleep disorders during their sleep, mainly obstructive sleep apnea syndrome and/ or insomnia.

Methodology: In a Group composed by 31 elements (Group 1), a questionnaire was filled out and in another Group composed by 38 elements (Group 2), besides the questionnaire being filled out; a cardio respiratory sleep test was carried out. All elements of the sample signed their informed approval and their anonymity was guaranteed.
\end{abstract}

Results: In Group 1 it was verified that the majority of the typical symptoms of the obstructive sleep apnea syndrome and insomnia appeared and aggravated at the beginning of peri or post menopause, thus, from this stage onwards they use a ventiloterapy treatment. In Group 2, 68.4\% of the women had signs of obstructive sleep apnea syndrome.

Conclusion: Peri and post menopause should be considered as a risk factor for the development of many sleep disorders, thus a larger exposure of these disorders should be made among health professionals and target population.

Keywords: peri menopause, post menopause, sleeps disorders, insomnia, obstructive sleep apnea syndrome
Volume I Issue 3 - 2014

\author{
Susana Margarida Oliveira Rodrigues,' Rosa \\ Maria Felix Fonseca ${ }^{2}$ \\ 'Cardiopneumologia, Escola Superior de Tecnologia da Sa \\ ${ }^{2}$ Cardiopneumologia, Portugal
}

Correspondence: Susana Margarida Oliveira Rodrigues, Cardiopneumologia, Escola Superior de Tecnologia da Saúde do Porto, Portugal, 4I05 124th AVE SE \# 1003 Bellevue,WA, 98006 USA, Tel 142587743 I3, Email s.oliveira.rodrigues@gmail.com

Received: June 15, 2014 | Published: July 18, 2014
Abbreviations: OSA, obstructive sleep apnea, URT, upper respiratory tract, $\mathrm{AHI}$, apnea hypopnea index, $\mathrm{SaO}_{2}$, arterial oxygen saturation, $\mathrm{FSH}$, follicle stimulating hormone, LH, luteinizing hormone, GABA, gamma aminobutyric acid, NREM, non rapid eye movements, REM, Rapid Eye Movements, SPSS, statistical package for the social sciences, SD, standard deviation, BMI, body mass index, HBP, high blood pressure, AASM, american academy of sleep medicine, H1, alternative hypothesis, H0, null hypothesis, CI, confidence interval

\section{Introduction}

Framing of topic

\section{Sleep now-a-days}

Sleep assumes anessential role in everyone's life quality. We spend approximately a third of our day sleeping and when there are signs of sleep disorders, it has a significant impact in the life quality of patients. ${ }^{1}$ In the last 30 years sleep medicine developed rapidly due to the high quantity of information obtained on the many disorders that affect sleep. Sleep disorders are defined as any modification that can affect, perturb or involve the sleep and there are frequent complaints among women that are in peri or post menopause. It is thought that the main sleep disorders that occur in peri- and post menopause are insomnia and obstructive sleep apnea syndrome (OSA). ${ }^{1-3}$

Insomnia and OSA: Insomnia is characterized by the difficulty in starting and/or maintain the sleep and/or by waking up before it's supposed to, leading to the presence of a non-restorative sleep. In this sense, sleep becomes insufficient for a good quality caution and physical and mental well-being during the day, which consequently, leads to a difficulty on performing daytime activities. The most frequent neuropsychiatry symptoms in menopause (irritability, anxiety, nerves, depression, fatigue, loss of concentration and memory) intensify in women that suffer insomnia. ${ }^{2-6}$ OSA is characterized by the presence of recurrent episodes of partial or total upper respiratory tract (URT), namely of the pharynx, during sleep. The typical symptoms associated to OSA are: snoring, sleepiness during the day and apneas. However, initially, women can present atypical symptoms (insomnia, fatigue, coexistent clinical depression or hypothyroidism) that can hide the real diagnosis. ${ }^{4,5,7-16}$

The basic respiratory events that characterize OSA are: apnea (deriving from the total obstruction of URT) and hypopnea (deriving from a partial obstruction of URT). These events are associated to micro-arousals, hypoxemia and, in cases of extended events the Apnea-hypopnea Index (AHI) that is defined by the number of apneas and/or hypopnea per hour of sleep, allowing to classify the gravity of OSA. Nevertheless, the determination of AHI isn't enough to classify the gravity of OSA, therefore it should also be related to the degree of sleepiness during the day and with to Arterial Oxygen Saturation $\left(\mathrm{SaO}_{2}\right)^{7-11,13,14,17}$

\section{Female climacteric, peri menopause, menopause and post menopause}

Female climacteric is a period with many physical and emotional transformations which the main phenomena is menopause, indicating 
the end of women's reproductive capability, with gradual decrease of ovarian and estrogenic functions. It's a continuous process and not a prompt stage of a woman's life, since it embodies peri and post menopause. Normally sleep quality deteriorates with the evolution of climactic. ${ }^{18-20}$ Peri menopausa (also known as "menopause transition") is the period that occurs since the irregular cycles start until one year after the last menstruation and it is associated with large hormonal variations: decrease of circulating estrogen and testosterone and an increase of follicle stimulating (FSH) and luteinizing hormones (LH). These hormonal changes are responsible for physical, physiological and psychological changes, which affect sleep. It is also at this stage that women present more symptoms, namely: asphyxia crisis, night sweats, dry skin and mucus and physiological changes (cognitive, intellectual and humor performance, anxiety and depression). Generally peri menopause appears between the ages of 45 and $50.4,18-23$

Natural menopause is gradual disappearing of spontaneous menstruation and the end of a fertile life for women. It is recognized at the end of 12 consecutive months without menstruation period, without being associated with other physiological and pathological changes. It occurs when follicles aren't enough to produce estrogen in necessary concentrations to induce a proliferation of the endometriumand become a menstruation. Therefore, estrogen starts becoming secreted in a lower quantity and women's organism is exposed to a new hormonal environment: hypoestrogenism. Generally it appears around the age of 50, even though the age can vary from women to women. ${ }^{2,19-23}$ Post menopause is a long period of a woman's life that occurs from the last year of her last menstruation until death and peri menopause is a period of approximately five years before menopause where there are regular menstrual cycles which become shorter and afterwards longer. ${ }^{20}$

\section{Main functions of estrogen and progesterone during sleep}

Progesterone besides being an important respiratory stimulator, also works as anxiolytic because it activates the benzodiazepines receivers, which in turn stimulates the gamma-aminobutyric acid receivers $(\mathrm{GABA})$. GABA is responsible for sleep without non-rapid eye movements (NREM). Estrogen is associated to the decrease of body temperature to lower levels, during sleep. This hormone also increases the total time of sleep, increases the duration of sleep cycles in a stage of rapid eye movements (REM), decreases sleep latency and the number of awakenings. This fact is verified when women in peri menopause increase two times the number of awakenings that occur, during the latency stage of the menstrual cycle, particularly when both hormones (estrogen and progesterone) present low levels. ${ }^{3,4}$

\section{Menopause, hormonal changes and sleep}

Many studies suggest that women who are in a fertile life transition to menopause and women that are in apost menopause present sleep difficulties, with a larger frequency than younger women that are in peri menopause. Some authors suggest that estrogens and progesterone carry out a protecting effect in the URT, warning women that they are in pre menopause of developing apnea during sleep. Progesterone and perhaps estrogen perform an important role in the regulation of the respiratory drive, increasing the answer to hypoxia ventilation and stimulating the reversion of hypoventilation. Thus, the reduction of those hormone's levels can predispose some women to presenting sleep respiratory disorders, due to the reduction of the drive action in URT that may lead to an imbalance between the strength that promotes the maintenance of its permeability and the strength that promotes its collapse, originating total of partial obstruction of URT. ${ }^{3,9,11,14,16,17,23-27}$

With the development of peri and post menopause, an intense hormonal variability, namely in the estrogen levels, that originates vasomotor symptoms (asphyxia crisis, heat, night sweats, among others) responsible for the frequent awakenings and/or microawakenings and that may lead to the appearance of different sleep disorders, being the most common insomnia and OSA. It is also after menopause that women have a larger probability of presenting overweight or obesity which is associated with chronic snoring and it constitutes a risk factor for OSA. Some studies refer that the reduction of estrogen and progesterone levels can influence the distribution of body fat, which can origin a larger disposition of fat in the abdominal region (Android obesity) and in URT, at a neck level, increasing the neck perimeter. However, other studies show that women's weight gain during menopause seems to be related, more intimately, with physiological and behavioral changes associated to ageing rather than estrogen deprivation. ${ }^{1-6,18-24,27-31}$

Anxiety and depression that can emerge at this stage in women's lives and it can also be one of the causes and not the consequence, of the changes during sleep, namely of insomnia. Hence, OSA and insomnia can be mistaken with a clinical framework of depression, aggravating the patient's clinical situation. During a treatment with antidepressants, some can also act as muscle relaxant which can contribute to the obstruction of URT. The decrease of estrogen level scan also be in the origin of anxiety and depression development. However, in addiction, generally women at this stage of their lives, overcome many changes, namely: social (period of changes to retirement), emotional (family "nest is empty", since normally at this stage children leave their parents' house) and a period to face ageing. This factors can also contribute to the development of depression and anxiety. The most frequent complaints from women that are going through this stage are: frequent night awakenings, difficulty in getting back to sleep and, sometimes difficulties in falling asleep. Yet, besides insomnia being more prevalent than OSA, this last one also assumes an important role due to the serious cardiovascular compilations that it may result. $1,2,4,6,18-24,26,28-30$

\section{Study substantiation}

The increase of life expectancy average of population, in general, due to the improvement of social conditions and to the advance of medical technology in these last year's, lead to a larger interest for the study of life quality in general and also in specific populations. One of those populations have been women, especially women in menopause. Otherwise from what happened in the past, where there were only few women that lived long periods after menopause, presently a large period of their lives are developed during this stage which is associated to a significant change of their state of health (whether in a psychological or physical point of view). The presence of insomnia and/or OSA during the transition to menopause and in post menopause causes significant disadvantages at a socio-labor level affecting women's life quality. Therefore, they should be well evaluated, treated and accompanied by health professionals for it to be quickly resolved $2,4,5,20,24$. The selection of the inherent theme for this research was based not only on the personal enthusiasm of the diagnostic area and accompaniment of sleep pathologies, but also based on the fact that sleep disorders are more and more an important problem in public health, having a significant impact in modern society's life. 


\section{Objective of study}

The central objective of this project is evaluate the changes in sleep characteristics in women that are in peri- and post menopause, as well as observe their vulnerability in presenting certain sleep pathologies, namely insomnia and OSA, at this stage of their lives.

\section{Methodology}

\section{Characterization and duration of study}

A prospective and transversal observational study was carried out since October 2009 until June 2010 after being approved by the managers of GASIN-Air Product Group.

\section{Inclusion and exclusion criteria of sample}

The inclusion criteria for each element that forms the sample were: being female, comprehend ages between 45 and 65 , being in peri or post menopause, having started a ventilotherapy after entering peri or post menopause (only in Group 1) and capability of reading and signing the informed consent. The exclusion criteria of the sample were: having carried out total hysterectomy, presence of severe dementia or any other severe psychological disorder, illiteracy, presently taking a hormonal substitution therapy and having stated a ventilotherapy before entering peri menopause or post menopause (only in Group 1).

\section{Selection of participants and dimension of study sample}

Taking into account the criteria mentioned above, a random sample was selected, constituted by 69 elements, with ages comprehended between 45 and 65 , resident in the northern region of Portugal. This sample was divided in two Group s. Group 1 was constituted with 31 female elements that were in peri or post menopause, had a previous OSA diagnosis with or without insomnia and prescription for ventilotherapy with the company GASIN-Air Products Group. Group 2 constituted by 38 elements, women who were also in peri and post menopauseand didn't know that they suffered from any of these sleep disorders. Therefore, it is obvious that the objectives in each Group are different. The objective of Group 1 was evaluating if the beginning of peri or post menopause triggered difficulties during the sleep and the consequent diagnosis of OSA with or without insomnia. In Group 2 the objective was to study the suspect of OSA and/or insomnia, as well as other sleep changes related to peri and post menopause.

\section{Instruments used to gather data}

To gather the information the following investigation instruments were used: two questionnaires, one for Group 1 and another one for Group 2, the Epworth scale and the cardiorespiratory sleep study carried out using the Stardust ${ }^{\circledR}$ II equipment, from Philips Respironics (property of Gasin-Air Products Group ).

\section{Gathering data process}

In Group 1 the gathering of data was carried out by filling in a questionnaire by all elements. In Group 2 the gathering of data was carried out by filling in a questionnaire and carrying out a cardiorespiratory sleep study at each element of the Group's home. So, in Group 1 the analysis of the results was merely subjective, once it was based on the answers of each element, whereas in Group 2 the evaluation was not subjective (because the answers from each element were taken into account), but also objective, based on a cardio respiratory sleep study and it's correspondent AHI. The participants answered the research protocol at their homes, in an isolated and silent space. The fulfillment took around 15 minutes.

\section{Ethical principles}

According to the Helsinquia statement for ethical principles. The use of questionnaires was preceded by a detailed oral and written explication, of the objectives of the present study, as well as the importance of the fulfillment of all items and, of its reading by patient, of the informed consent, signing it in case the use of dada was authorized to carry out the study. All questions that emerged from the questionnaire were clarified without having any influence in the respective answers by the author of the study. The informed consent was signed by all women that participated in this study, guaranteeing the anonymity and confidentiality of their data in each and every moment of the study.

All the people that were part of the sample studied had the physical and mental conditions to sign and duly understand the informed consent. It was always assured the respect for autonomy and human dignity and promoted the physical and mental well-being of participants. All the elements of the sample were informed of the right to participate or not in the study and the power to withdraw their consent at any moment, without being exposed to reprisals.

\section{Explanation of the instruments used to gather the information}

Questionnaire: The questions that made this questionnaire were selected taking into account the variables necessary for a future analysis of the subject being studied. Hence, the questionnaire used was carried out taking into account the literature information's about menopause and the main sleep disorder symptoms to be studied (insomnia and OSA). However, the analysis of other questionnaires had to be taken into account namely: the domain of the questionnaire developed by Souza and Aldrighi and the questions made in "Sleep Evaluation Questionnaire", in "Cuestionario Oviedo delSueño" and in"Berlin Questionnaire (for Sleep Apnea)". ${ }^{1,18}$ Nevertheless, the questionnaires mentioned before were only used to make it easier to build some questions, since the selection of questions, the order and the way the questions were made were changed and adapted better to the objectives of this study. The questionnaire used in this study to gather data is divided in three parts: the first part consists of 24 questions in total which are divided in five Group s: personal characterization, life habits, health situation, menopause and sleep quality, the second part consists of a chart (Table 1) with 9 questions which aim for a sleep quality comparison before peri- and post menopause with the sleep quality after entering peri and post menopause, and finally, the third part consists of an Epworth somnolence scale.

Table I Distribution of frequencies according to age and BMI

\begin{tabular}{lllll}
\hline & Average & SD & Minimum & Maximum \\
\hline Group I & & & & \\
Age (years) & 56,9 & 6,9 & 40 & 65 \\
BMI (kg/m2) & 30,3 & 7,2 & 19,5 & 48,1 \\
Group 2 & & & & \\
Age (years) & 55,4 & 5,4 & 46 & 65 \\
BMl (kg/m2) & 25,9 & 3,2 & 21,6 & 35,5 \\
\hline
\end{tabular}


The differences between the questionnaires for Group 1 and Group 2 are minimum, only changing the way the questions are made because in Group 1 the objective is not that women characterize the sleep they presently have, since they're using Ventilotherapy they shouldn't have any difficulties during the sleep, but the objective is taking into account the sleep quality and the symptoms after entering peri and post menopause before using ventilotherapy. It was carried out a test-retest of the answers given by the 30 women that fulfilled the same characteristic of the sample, to confirm the reproducibility and reliability of the questionnaire used.

\section{Cardiorespiratory sleep study}

To carry out the sleep studies at home it was used acardiorespiratory polygraph Stardust ${ }^{\circledR}$ II of Philips Respironics (and respective analysis software), which allowed the registry of the following parameters: $\mathrm{SaO}_{2}$, cardiac frequency, oro-nasal flow, respiratory effort, body position and snoring. After undertaking each exam, a download was made to the computer and they were all analyzed. It is important to refer that all the studies were supervised and reviewed by the supervisor of this research project.

\section{Statistical analysis}

With the computer program Statistical Package for the Social Sciences (SPSS 16.0) a statistical treatment was made with all the gathered data. Some of the data was analyzed in a descriptive statistical form, calculating the distribution frequencies, averages, standard deviation (SD) and maximum and minimum standards. The different averages were compared using some tests: test $\mathrm{T}$ for collected samples, Chi-square test for independence, Binomial test and Wilcoxon test. For alpha $(\alpha)$ it was defined a value of 0.05 , thus all values $p$ lower than 0.05 allow significant statistical conclusions.

Variables being studied: The variables being studied are divided as:

i. Independents are Group $\mathrm{Ed}$ in the following categories: socio-demographic (age, marital status, profession, working schedule), behavioral (physical exercise, alcohol and/or caffeine consumption, smoking habits and use of medication to sleep) and those regarding medical conditions (body mass index (BMI), diabetes, high blood pressure (HBP), hypercholesterolemia, among others).

ii. Dependents are variables regarding sleep quality before and after entering peri- and post menopausehours they sleep and slept per night, time they take and took to fall asleep, AHI and the daytime somnolence degree evaluated according to the Epworth scale.

\section{Operation of variables}

To classify if a woman was in peri or in post menopause the following factors were taken into account, age, presence of irregular menstrual cycles or absence of menstrual cycles and the date of the last menstruation. The "ethnic" variable was quantified according to the following possibility of answers: White, African, Oriental, Indian, Gypsy, Mixed and others. In the case of the "marital status" variable the possibility of answers were: single, married/life partners, separated/ divorced, widow and others. The "working schedule" variable was evaluated taking into account the following categories: daytime, nighttime and by shifts. The variables regarding smoking habits, alcohol and/or caffeine consumption, practicing physical exercise and taking medication to sleep, were only evaluated taking into account the affirmative or negative answer to the respective questions. Regarding the "known illnesses" variable, its analysis also took into account the respective answer possibilities: HBP, hypercholesterolemia, diabetes and others. To evaluate the "awakening before your suppose to" variable, affirmative and negative answers were quantified and the time (in hours) that they woke up before they were supposed to. All variables mentioned in chart 1 were analyzed according to the following frequency evaluation chart: never, rarely (once or twice a week), frequently (three to four times a week) and very frequently (five to seven times a week).

To proceed to the BMI calculation, it was considered the reason between the body mass total, in kilograms and the square of height in meters $\left(\mathrm{kg} / \mathrm{m}^{2}\right)$. The classification followed the World Health Organization standards: $\leq 18.5 \mathrm{~kg} / \mathrm{m}^{2}$ (weight below normal), 18.5 to $24.9 \mathrm{~kg} / \mathrm{m}^{2}$ (normal weight), 25 to $29.9 \mathrm{~kg} / \mathrm{m}^{2}$ (overweight), 30 to $34.9 \mathrm{~kg} / \mathrm{m}^{2}$ (obesity degree I), 35 to 39.9 (obesity degree II) and $\geq 40 \mathrm{~kg} /$ $\mathrm{m}^{2}$ (obesity degree III or morbid obesity). ${ }^{32}$ The Epworth somnolence scale is composed by eight situations and, for each one of them, the patients have to classify the probability of falling asleep on a scale from 0-3 (none, unlikely, moderated and great probability). The sum of all the questions can vary from $0-24$, being considered the existence of a somnolence pathology when the sum is higher than 10 and that there's a high degree of hypersomnia when the sum is higher than $16{ }^{7,9,13}$

The cardiorespiratory sleep studies were analyzed taking into account the criteria suggested by American Academy of Sleep Medicine. ${ }^{33}$ Therefore, it was considered that there was an apnea when there was a total cease or $90 \%$ decrease or more of airflow during sleep, with duration equal or superior to 10 seconds. Most of the classified apneas were an obstructive type (there's a respiratory effort during the event), even though there may also exist in a variable portion, central type of events (there is no respiratory effort during the event) and mixed (there is no respiratory effort at the beginning of the event, subsequently reappearing after anoro-nasal flux is reestablished). To classify a hyponea it would have to be verified, during at least 10 seconds, a reduction of $50 \%$ or more of oro-nasal flux associated to a desaturation of $\mathrm{SaO}_{2}$ of at least $3 \%$ or a reduction of oro-nasal flux superior to $30 \%$ associated to a desaturation of at least $4 \%$. AHI was calculated for the elements of Group 2, with the objective of classifying the OSA gravity: slight when AHI is situated between 5 and 14.9, moderated when AHI is situated between 15 and 29.9 and severe when AHI is superior than $30 .^{7-14,17,27}$

\section{Study hypotheses}

To reach the main purpose of this study, many hypotheses were formulated to test the relation between the variables. Only the alternative hypotheses $(\mathrm{AH})$ will be mentioned, because the null hypotheses ( $\mathrm{H} 0)$ are for all tests carried out the opposite of the respective H1. The common alternative hypotheses of Group s1 and 2 were the following:

1. The average of the differences between the number ofhours that they slept per night before entering peri and post menopause and the number ofhours that they sleep after entering peri- and post menopause is different from zero.

2. The average of the differences between time (in minutes) that they took to fall asleep before entering peri- and post menopause and the time (in minutes) that they took to fall asleep after entering peri and post menopause is different from zero. 
3. The proportion of women that wake up before they're supposed to is different from the proportion of women that don't wake up before they're supposed to.

4. The BMI is dependent of the peri- and post menopause.

5. For all the questions made in chart 1 of the questionnaire, the hypothesis for each one was: there are differences between before and after peri- and post menopause.

The alternative hypotheses for Group 2 were:

1. The average of the differences between AHI in supine position and $\mathrm{AHI}$ in non-supine position is different from zero.

2. AHI is dependent from age.

3. AHI is dependent from BMI.

4. AHI is dependent from caffeine consumption.

5. AHI is dependent from alcohol consumption.

6. AHI is dependent from practicing physical exercise.

7. AHI is dependent from medication to sleep.

8. $\mathrm{AHI}$ is dependent from peri- and post menopause.

\section{Results}

\section{Characterization of sample}

The characterization of the sample studied is presented in a resumed way in the Table 1 and 2. In Table 1 it is verified that the average of the ages in Group 1 was $56.9 \pm 6.9$ and in Group 2 5.4 5.4. As in the BMI, the average of Group 1 was $30.3 \pm 7.2 \mathrm{~kg} / \mathrm{m}^{2}$ and Group $225.9 \pm 3.2 \mathrm{~kg} / \mathrm{m}^{2}$. In Table 2 it is verified that Group 1 was formed by $9(29 \%)$ women that were in peri menopause and $22(71 \%)$ in post menopause and in Group 2, 8 (21.1\%) were in peri menopause and 30 $(78.9 \%)$ in post menopause. All the elements of the sample were of Caucasian ethnicity.

Table 2 Distribution (in percentage) of menopause variables, work schedule and BMI

\begin{tabular}{lll}
\hline & Group I (\%) & Group 2 (\%) \\
\hline Menopause & & \\
Peri menopause & 29 & 21,1 \\
Post menopause & 71 & 78,9 \\
Working Schedule & & \\
Day time & 32,3 & 26,3 \\
Night time & & \\
By shifts & 3,2 & 13,2 \\
Retired/Domestic & 64,5 & 60,5 \\
BMI & & \\
Normal weight & 22,6 & 39,5 \\
Overweight & 35,4 & 55,3 \\
Obesity degree I & 22,6 & 2,6 \\
Obesity degree II & 9,7 & 2,6 \\
\hline
\end{tabular}

In Group 1, 67.7\% were married, $19.3 \%$ widow, $6.5 \%$ single and $6.5 \%$ divorced. As for the marital status in Group 2, 81.6\% were married, $10.5 \%$ widow and $7.9 \%$ were divorced. In Group 1 and in
Group 2, the majority of the elements had 4 th grade, $32.3 \%$ and $50 \%$, respectively, and only $6.5 \%$ and $10.5 \%$ had a degree. The sample was formed mainly by retired and domestic women $(64.5 \%$ in Group 1 and $60.5 \%$ in Group 2). Among the women that were still professionally active, none of them had a nighttime shift, having the majority a daytime shift (32.3\% in Group 1 and 26.3\%, in Group 2). In Group 1 the majority had overweight (35.4\%), the percentage of women with normal weight was equal to the percentage of women with obesity degree I (22.6\%) and the same happens regarding the percentage of women with obesity degree II and obesity degree III (9.7\%). In Group 2 , the majority also had overweight $(55.3 \%), 39.5 \%$ had normal weight and the percentage of women with obesity degree I was equal to the percentage of women with obesity degree II $(2.6 \%)$, there was no women with obesity III in this Group. With the Chi-square test for Independence, Group s1 and 2 obtained a $p=0.445$ and $p=0.737$, respectively, superior to $0.05(\alpha)$, thus in this case $\mathrm{H} 0$ could not be rejected, there was no significant statistical evidence to affirm that the BMI was associated with peri and post menopause.

\section{Subjective sleep quality}

In Group s 1 and $2,54.8 \%$ and $47.4 \%$ of the women, respectively, affirm that they sleep lesshours after they entered peri and post menopause. To analyze if that difference was statistically significant, a $\mathrm{T}$ test for gathered samples was carried out and showed that there were significant statistical evidences $(p \approx 0.00)$ to affirm that the difference of average between the number of hours/night they slept before and after entering peri and post menopause is different from zero (H0 was rejected in this test, because the value of $p$ is inferior to 0.05 ). With this test it was verified, that in Group 1, women after entering peri and post menopause slept, in average at least $1.5 \pm 1.3$ hours/night and, in Group 2, they slept at least $1.3 \pm 0.92$ hours per night. It can be affirmed, with a confidence interval (CI) of $95 \%$ that women in peri and post menopause belonging to the population in general, slept between 0.7 and 1.8 hours/night less after entering peri and post menopause.

In Group s1 and 2, 35.5\% and 31.6\% of the women, respectively, referred spending too much time falling asleep after being in peri and post menopause. To verify if this difference is statistically significant it was also carried out a $\mathrm{T}$ test for gathered samples. Analyzing the test, it was concluded that the average of the difference between the time (in minutes) that took to fall asleep before and after entering peri and post menopause is different from zero ( $\mathrm{H} 0$ is rejected in this case because $p=0.013$ in Group 1 and $p=0.036$ in Group 2). In Group 1 women took, in average, more $21 \pm 9$ minutes to fall asleep after entering peri- and post menopause, and in Group 2 they took more $13 \pm 5$ minutes. With a confidence interval of $95 \%$, it can be concluded that women in peri and post menopause belonging to population in general took between 2 and 16minutes more to fall asleep after entering peri- and post menopause. In Group s1 and 2,35.5\% and $60.5 \%$ of the women affirmed waking up before they're supposed to. In Group 1, from the women that woke up before they were supposed to, $45.4 \%$ woke up 2 hours before, $27.3 \%$ woke up 1 hour before and $27.3 \%$ woke up half an hour before. In Group 2: $47.8 \%$ woke up one hour before, $26.1 \%$ woke up 2 hours before, $21.7 \%$ woke up half an hour before and $4.4 \%$ woke up 3 hours before.

A Binomial test was carried out and it was verified that the proportion of women that wake up before they're supposed to is different from the proportion of women that don't wake up before they're supposed to. For Group 1 we obtained a $p=0.150$ and for Group 2 a $p=0.256$. H0 was not rejected in this case, because there are no existence of significant statistical evidences to affirm that in the population of women in peri or post menopause, the proportion of 
those who woke up before they were supposed to was different from the proportion of those that did not wake up before they were supposed to. In Table 3 the results obtained can be analyzed with a Wilcoxon test. The objective of this test was to evaluate if the frequency of symptoms regarding the subjective sleep quality increased, decreased or did not change after the beginning of peri or post menopause.

Table 3 Comparison of frequency when symptoms occurred in women from Groups I and 2 before and after peri and post menopause

\begin{tabular}{|c|c|c|c|c|c|c|c|c|c|}
\hline \multicolumn{2}{|c|}{ S. No } & \multicolumn{4}{|c|}{ Group I } & \multicolumn{4}{|c|}{ Group 2} \\
\hline & & $\mathrm{I} *$ & $2^{* *}$ & $3 * * *$ & Value $p$ & $I^{*}$ & $2^{* *}$ & $3 * * *$ & Value $\mathrm{p}$ \\
\hline & & $(\%)$ & $(\%)$ & (\%) & & $(\%)$ & $(\%)$ & (\%) & \\
\hline I & Waking up during the night & 6 & 65 & 29 & 0 & & 55 & 45 & 0 \\
\hline 2 & Waking up with shortness of breath & & 58 & 42 & 0 & & 11 & 89 & 0.125 \\
\hline 3 & Stop breathing during the night & 6 & 46 & 48 & 0.001 & 2 & $\mathrm{II}$ & 87 & 0.375 \\
\hline 4 & Snoring during the night & & 52 & 48 & 0 & 3 & 34 & 63 & 0.001 \\
\hline 5 & Waking up with the feeling they didn't rest & 3 & 52 & 45 & 0 & & 37 & 63 & 0,00 \\
\hline 6 & Waking up with headaches & 3 & 52 & 45 & 0 & & 26 & 74 & 0,002 \\
\hline 7 & Fatigue/Somnolence during the day & 3 & 58 & 39 & 0 & & 42 & 58 & 0,00 \\
\hline 8 & Sudden sensations of sleepiness during the day & 3 & 45 & 52 & 0,001 & 2 & 22 & 76 & 0,023 \\
\hline 9 & Harmful socio-labor relationships for not sleeping well & & 32 & 68 & 0.002 & & 11 & 89 & 0,125 \\
\hline
\end{tabular}

*After entering peri- and postmenopause it happened less than after peri and postmenopause

**After entering peri- and postmenopause it happened more often than before peri and postmenopause

***There was no difference in the frequency of symptoms before and after entering peri and postmenopause

In Group 1 the value of $p$ was always inferior to 0.05 ; consequently $\mathrm{H} 0$ can be rejected, since there were always significant statistical evidences to affirm that there were differences in the frequency of the symptoms between "before" and "after" peri and post menopause. The symptoms that started occurring with more frequency after peri and post menopause were: waking up during the night (in $65 \%$ of women), waking up with shortness of breath (in $58 \%$ of women), snoring during the night (in $52 \%$ of women), waking up with the feeling they didn't rest (in 52\% of women), waking up with headaches (in $52 \%$ of women) and feeling fatigue/somnolence during the day (in $58 \%$ of women). For the rest of the symptoms, women of the sample did not feel differences after entering peri and post menopause.

In Group 2, the only symptom that increased its frequency after peri and post menopause was waking up during the night (in 55\% of women), with a $p$ value inferior to 0.05 ( $\mathrm{H} 0$ was rejected), from what can be affirmed that the difference obtained was statistically significant. As for the remaining symptoms, the women from this Group did not feel the difference of its occurrence after entering peri and post menopause. However, it is important to highlight that for the symptoms: waking up with shortness of breath, stop breathing during the night and harmful socio-labor relations for not sleeping well, the $p$ value obtained was superior to 0.05 , therefore $\mathrm{H} 0$ could not be rejected, this means, it couldn't be concluded if there was existence of significant statistical differences between "before" and "after" peri and post menopause for those symptoms.

\section{Objective analysis of sleep quality (Group 2)}

The cardiorespiratory sleep studies allowed to quantify the AHI in Group 2. As it was verified in Figure 1, 68.4\% of women in Group 2 had AHI superior to 5 per hour (evidence of OSA) and 31.6\% had AHI inferior to 5 per hour (without evidence of OSA). In Figure 2 it can be observed that, the ones that had OSA, $80.8 \%$ had a mild degree, $15.4 \%$ moderate and $3.8 \%$ severe. In Table 4 it can be verified that the average of AHI was $9.1 \pm 6.75$ events/hour, varying between 1.50 , minimum and 36.2, maximum. As for the snoring index, the average was $108.5 \pm 126.1$ events/hour and the minimum and maximum values registered were 1.2 and 553.4 events/hour, respectively. The AHI registered in supine position was, in average, superior to the registry in non-supine position (15.3events/hour comparatively to 6.3events/ hour). Therefore, according to the differences observed between the $\mathrm{AHI}$ in the supine and non-supine positions, a $\mathrm{T}$ test for gathered samples was carried out, to verify if the differences were statistically significant. With the analysis of the test, it was verified that $\mathrm{H} 0$ $(p \approx 0.00)$ could be rejected, since there were significant statistical evidences that AHI in supine position is superior to the non-supine position. It was also verified that in the supine position it occurred, in average, more 8.7 events/hour than in the non-supine position. We can also affirm, with the confidence interval of $95 \%$, that the difference between $\mathrm{AHI}$ in supine position and non-position, in the population of women in peri and post menopause, would be between 5.3 and 12.1 events/hour.

Table 4 Distribution of frequencies according to $\mathrm{AHI}, \mathrm{AHI}$ in supine position and non-supine position and snoring index

\begin{tabular}{lllll}
\hline & Average & SD & Minimum & Maximum \\
\hline AHI (events/hour) & 9,1 & 6,75 & 1,50 & 36,2 \\
Snoring Index (events/hour) & 108,5 & 126,1 & 1,2 & 553,4 \\
AHI Supine (events/hour) & 15,3 & 12,7 & & 56,4 \\
AHI Non-supine (events/hour) & 6,3 & 5,6 & & 28,1 \\
\hline
\end{tabular}

Citation: Rodrigues SMO, Fonsec RMF. Menopause and sleep disorders: does peri and post menopause influence the development of OSA and/or insomnia? J Cardiol Curr Res. 2014;I(3):8I-9I. DOI: 10.15406/jccr.2014.01.00017 


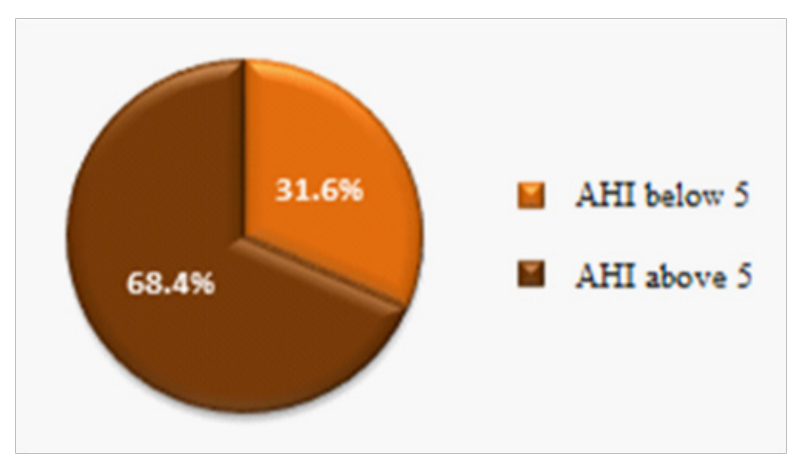

Figure I Distribution of elements in Group 2 according to AHI.

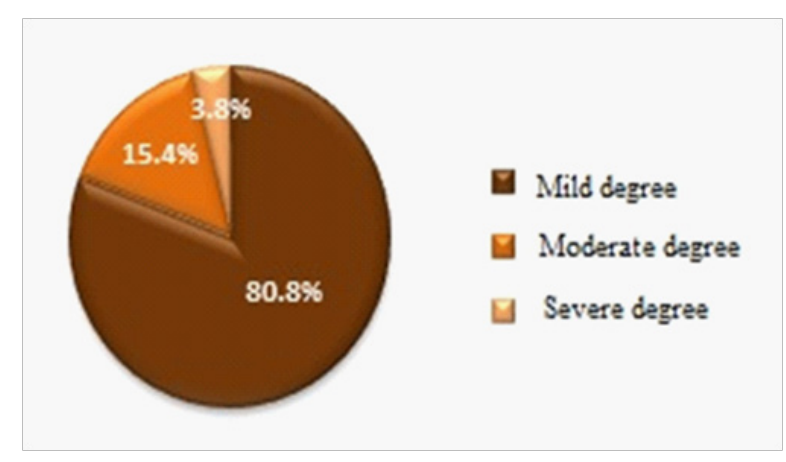

Figure 2 Distribution of severity degrees of OSA according to AHI.

Table 6 Association between severe degree of OSA and weight (classified according to BMI)

\begin{tabular}{lllll}
\hline & Normal weight (\%) & Over weight (\%) & Obesity degree I (\%) & Obesity degree II (\%) \\
\hline Without OSA & $53,3(n=8)$ & $14,3(n=3)$ & $100(n=1)$ & \\
Slight degree of OSA & $46,7(n=7)$ & $66,7(n=14)$ & & \\
Moderated degree of OSA & $19(n=4)$ & $100(n=I)$ \\
Severe degree of OSA & & & \\
\hline
\end{tabular}

In Figure 3 we can observe that among the women with diagnosed high blood pressure (HBP), only $14.3 \%$ didn't have any evidence of OSA (AHI inferior to 5/hour) and $85.7 \%$ had AHI superior to 5/ hour (57.1\% with AHI between 5 and 14.9/hour and 28.6\% with AHI between 15 and 29.9/hour). In Figure 4 we can verify that a similar situation also occurs with those that had hypercholesterolemia: $66.7 \%$ had AHI superior to 5/hour and $33.3 \%$ presented AHI inferior to 5/ hour. Within the elements that belong to Group 2, that didn't have any known illnesses: $73.4 \%$ presented AHI superior to $5 /$ hour and $26.6 \%$ evidenced AHI superior to 5/hour.

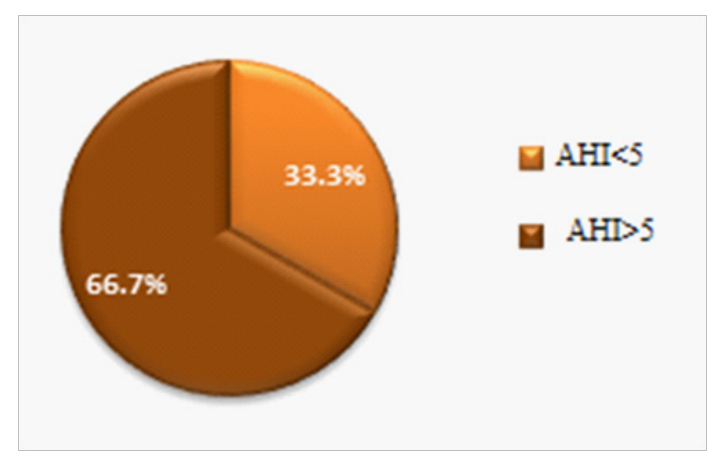

Figure 3 Association between the existence of $\mathrm{HBP}$ and $\mathrm{AHI}$.
As it can be verified in Table 5, the majority of women that belong to Group 2 were in menopause and had a AHI superior than 5/hour $(60.5 \%)$. Within the 8 women that were in peri menopause, 3 presented AHI superior than $5 /$ hour and 30 of which were in post menopause 23 presented AHI superior than 5/hour. None of the women that were in peri menopause presented AHI between 15 and 29.9/hour (suggestive of moderated OSA) or superior than 30/hour (evidence of severe OSA). As for the women that were in post menopause, 4 of them presented AHI between 15 and 29.9/hour and another possessed AHI superior than 30/hour. In Table 6 it can be verified that among the women that had normal weight, 8 of them $(53.3 \%)$ there was no evidence of OSA and in $7(46.7 \%)$ the analysis of the exam was suggestive of slight degree of OSA. Among the women that presented overweight, $14(66.7 \%)$ evidenced a slight degree of OSA, $4(19 \%)$ a moderated degree of OSA and $3(14.3 \%)$ there was no evidence of OSA. Only one woman with obesity degree I didn't have evidence of OSA and the only woman with obesity degree II presented a severe degree of OSA.

Table 5 Association between $\mathrm{AHI}$ and the perimenopause and postmenopause variables

\begin{tabular}{lll}
\hline & AHI $<5 /$ hour & AHI $>5 /$ hour \\
\hline Menopause & & \\
Perimenopause (\%) & $13,2(n=5)$ & $7,9(n=3)$ \\
Postmenopause (\%) & $18,4(n=7)$ & $60,5(n=2$ \\
\hline
\end{tabular}

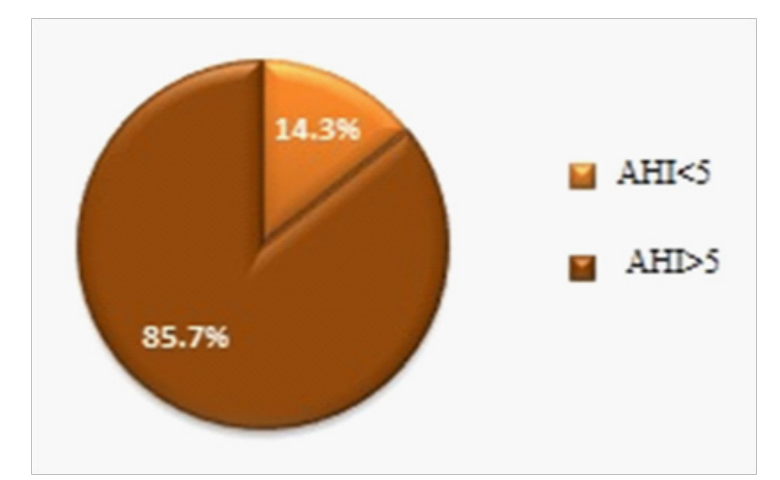

Figure 4 Association between the presence of hypercholesterolemia and $\mathrm{AHI}$.

In Table 7 it can be observed that among the women that affirmed taking medication to sleep $80 \%$ had an AHI superior to 5/hour and only $20 \%$ had AHI interior to 5/hour. As for those who stated not taking medication to sleep $66.7 \%$ had AHI superior to $5 /$ hour and $33.3 \%$ had AHI interior to 5/hour. As it can be verified in Table 8, the Epworth scale only revealed evidence of somnolence pathology in $10.5 \%$ of the cases that effectively presented OSA (slight degree). Being that in the remaining cases where there was evidence of OSA $(44.8 \%$ slight degree, $10.5 \%$ moderated and $2.6 \%$ severe) the Epworth scale 
was not sensible to pathology somnolence. Finally, the Chi-square test for independence was carried out to verify if there was or not any significant statistical connection between AHI and the different variables. In the sample investigated, in the majority of the tests carried out, $\mathrm{H} 0$ could not be rejected (values obtained for $p$ were superior to 0.05 ), so there were no significant statistical evidences to affirm that AHI was dependent of the following variables: $\operatorname{age}(p=0.681)$, Tabaco $(p=1.0)$, caffeine $(p=1.0)$, alcohol $(p=0.452)$, physical exercise $(p=1.0)$, medication to sleep $(p=0,682)$ and Epworth scale $(p=0,618)$. The variables that are significantly associated with $\mathrm{AHI}$, this is, that are dependent to $\mathrm{AHI}(\mathrm{H} 0$ rejected) are: $\mathrm{BMI}(p=0.003)$ and being in peri- or post menopause $(p \approx 0,00)$.

Table 7 Association between $\mathrm{AHI}$ and medication to sleep variable

\begin{tabular}{ll}
\hline \multicolumn{3}{l}{ AHI $<5 /$ hour (\%) } & AHI >5/hour (\%) \\
\hline Medication to Sleep & \\
Yes $\quad 20$ & 80 \\
No $\quad 33,3$ & 66,7 \\
\hline
\end{tabular}

Table 8 Association between the OSA gravity and the evidence of somnolence pathology by the Epworth Scale

\begin{tabular}{lll}
\hline & $\begin{array}{l}\text { Epworth scale } \\
\text { without evidence of } \\
\text { Somnolence pathology (\%) }\end{array}$ & $\begin{array}{l}\text { Evidence of } \\
\text { Somnolence pathology (\%) }\end{array}$ \\
\hline Without apnea & 21,1 & 10,5 \\
Apnea with slight degree & 44,8 & 10,5 \\
Apnea with moderated degree & 10,5 & \\
Apnea with severe degree & 2,6 & \\
\hline
\end{tabular}

\section{Discussion}

The assessment that sleep quality is deteriorated with peri and post menopause, confirms the data promoted in the lecture on this topic. The results obtained in this study prove that women, after entering peri and post menopause, start presenting less sleep quality, increasing symptomology inherent to insomnia, namely: $54.8 \%$ and $47.4 \%$ (in Group s 1 and 2) sleep two hours less per night, 35.5\% and $31.6 \%$ (in Group s 1 and 2) present more difficulties to fall asleep (at the beginning) and $35.5 \%$ and $60.5 \%$, in Group s 1 and 2, wake up earlier before they were supposed to (morning insomnia). In Groups 1 and $2,65 \%$ and $55 \%$ of women, respectively, felt they started to wake up with more frequency during the night, after entering peri and post menopause (difficulties to maintain sleep continuity). As a consequence of these results, there are many studies that demonstrate, that women after entering peri and post menopause refer more frequently symptoms suggestive to insomnia, being the most common: difficulty in falling asleep and to maintain sleep continuity, as well as morning insomnia. $6,20,23$

An epidemiological study quoted by Campos et al., 5 \& Soares ${ }^{24}$ revealed that the occurrence of insomnia among women with more than 30 years old varies from $26 \%$ to $45 \%$ and increase to values between $28 \%$ and $63 \%$ after menopause. Shin et al., ${ }^{34}$ carried out a study with 2400 mid-age Korean women and concluded that the prevalence of insomnia was more frequent in peri- and post menopause, comparing to pre menopause. Only $7.3 \%$ of women in pre menopause had insomnia, while women in peri- and post menopause the prevalence was of $15.9 \%$ and $19.7 \%$, respectively. As for the prevalence of difficulty in falling asleep it was 3.9\% within women in pre menopause, $9.0 \%$ within women in peri and $10.5 \%$ within women in post menopause. The difficulty to maintain the sleep was observed in $4.9 \%$ of women in pre menopause and in $10,5 \%$ and $13 \%$ of women in peri- and post menopause, respectively. Among the women that were in pre menopause, $3.0 \%$ affirmed waking up before they were supposed to while women in peri menopause and post menopause, the prevalence was of $7.2 \%$ and $11.5 \%$, respectively. ${ }^{34}$ In this study it was observed that in Group 1, women referred that the most suggestive
OSA symptoms started aggravating when they entered peri and post menopause: they snored more frequently $(52 \%)$, they started waking up more frequently with shortness of breath $(58 \%)$, with the feeling they didn't rest $(52 \%)$ and with headaches $(52 \%)$ and also started feeling more frequently fatigue/somnolence during the day $(58 \%)$. These results prove that, effectively, women in Group 1 started having significant OSA complaints, after entering peri and post menopause. After this period, most of them searched specialized help and started ventilotherapy.

In Group 2 the same situation wasn't verified, since women in this Group report that they didn't feel any difference regarding the frequency of these typical OSA symptoms, after entering peri and post menopause. The discrepancy in the symptomatic recognition between both Group s can be related with the fact that elements of both Group s had different comparing terms. While elements in Group 1 know have a more repairing sleep, they easily identify the non-normality to certain symptoms. As for elements in Group 2 they undervalue us because they don't have a comparing term. By means of AHI quantification, it can be concluded that in Group 2, 68.4\% of the women had AHI superior to 5/hour (suggestive of OSA) and only $31.6 \%$ of women from this Group presented AHI interior to 5/hour. From the total of elements that had AHI superior to 5/hour: in $80.8 \%$ there was an evidence of a slight degree of OSA (AHI between 5 and 14.9), in 15.4\% moderated degree (AHI between 15 and 29.9) and in $3.8 \%$ severe degree (AHI superior to 30 ).

In this study it was also verified that, from women that had AHI superior to $5 /$ hour, the majority were in post menopause $(60.5 \%)$ and only $7.9 \%$ were in peri menopause. None of the women that were in peri menopause presented AHI suggestive of moderated or severe OSA, while women that were in post menopause, 4 presented evidences of moderated OSA and possessed a suggestive AHI of severe OSA. With this data it can be concluded, in this sample, those women that were in post menopause had more susceptibility of presenting OSA.

These results meet the studies that have been already carried out by others which affirm a higher prevalence of OSA among women in peri and post menopause, comparing to women in pre menopause. 
Young ${ }^{35}$ carried out polysomnolographies to 1000 women between ages 20 and 100 and found a higher prevalence of respiratory sleep disorder in women in post menopause, comparatively with those in pre menopause. Anttalainen et al., ${ }^{30}$ concluded that, during the night, the respiratory sleep disorder occurred in larger proportions in women that were in post menopause, comparing to women in pre menopause (68.1\% versus $35.8 \%$, respectively). Danceyet al. ${ }^{17}$ also came to the same conclusion $(47 \%$ of women in post menopause suffered OSA, comparing to $21 \%$ in pre menopause). Bixleret al. ${ }^{25}$ in its study obtained an OSA prevalence in women in pre menopause inferior to the one found in women in post menopause, $0.6 \%$ and $3.9 \%$, respectively. In a posterior study carried out by Yong et al., ${ }^{27}$ it was verified prevalence in sleep disorder was inferior in women in pre menopause $(10.8 \%)$, regarding women in peri and post menopause (18,4\% and $29,1 \%$, respectively).

The studied sample was made, in its majority, by women with more weight than normal (77.4\% in Group 1 and $60.5 \%$ in Group 2). In Group 1, 35.4\% presented overweight, $22.6 \%$ obesity degree I, 9.7\% obesity degree II and $9.7 \%$ obesity degree III and in Group $2,55.3 \%$ had overweight, $2.6 \%$ obesity degree I and $2.6 \%$ obesity degree II. In Group 2, it was also observed, that women with normal weight, the majority didn't have OSA (53.3\%) and among women with overweight, $85.7 \%$ presented AHI superior to $5 /$ hour (suggestive of OSA). It can also be verified that the only element in Group 2 with OSA severe degree presented an obesity degree II. These results are in conformity with the lecture, which refer that peri- and post menopause can be associated with increase of weight, which leads to an increase of prevalence and OSA gravity, for reasons that still aren't clarified. $^{2-4,17,20,27,28}$

Regarding the known illnesses, it was particularly interesting to observe that the results obtained in this study, for the association between HBP and AHI. In elements of Group 2 that presented HBP diagnosed, $85.7 \%$ had AHI superior to $5 /$ hour (evidence of OSA) and, only $14.3 \%$ of women with HBP had an AHI inferior to 5/hour (absence of OSA). Taking into account the association between HBP and OSA severity, it was verified that $57.1 \%$ of women with HBP had AHI between 5 and 14.9 (suggestive of OSA slight degree) and $28.6 \%$ of the elements that had HBP had an AHI between 15 and 29.9 (evidence of OSA moderated degree). These values that were obtained are in conformity with the study effectuated by Boleo-Tome et al. ${ }^{13}$ where it is verified that the prevalence of HBP increased with the gravity of OSA, but even in slight degrees (AHI between 5 and 14.9) the prevalence found was important $(52,5 \%)$. Other studies conclude that, approximately, $50 \%$ of the patients with HBP have OSA. ${ }^{11,13}$

In this study it was verified in a Epworth scale that it revealed a low sensibility to identify the presence of somnolence pathology in elements of Group 2, because, only in $21.1 \%$ the sum was inferior to 10 (absence of somnolence pathology) when effectively there no evidence of OSA and, only in $10.5 \%$ of the cases where the sum was superior to 10 (somnolence pathology) the evidence of OSA was present. In the remaining suggestive cases of OSA (44.8\% slight degree, $10.5 \%$ moderated degree and 2.6\% severe degree), the sum in the Epworth scale was inferior to 10 (absence of somnolence pathology). However, these facts make me reflect that the problem may not be in the scale itself, but in the accented symptomatic devaluation from elements of Group 2 regarding the bad quality of sleep. In the study carried out by Rosenthal and Dolan it was also verified that the Epworth somnolence scale had low sensibility (66\%) in the identification of OSA when AHI is equal to $5 /$ hour. $^{36}$
Finally, during the execution of this project, there was no interference of no systematic error nor mistake. In the meantime, I must refer that, when the first elements of Group 1 were inquired on the period of peri and post menopause that occurred at the beginning of ventilotherapy, some confusion was generated with the perception of the present sleep quality. As corrective action all the explications on the pretended objective were reinforced, in order to obtain clarity in the answers. That said, the internal existence can be measured throughout the study, taking into account the following factors: objectivity and randomness in the choice of the sample, gathering of data directly next to the elements of the sample and precision of statistic treatment and in the analysis of different results of interest. As for the external validity, probably the results obtained cannot be extrapolated in an accurate way for the population of all women that are going through peri and post menopause, because the majority of the data sample was collected in a rural region and only in the area of northern Portugal. The main limitations of this project were: the transversal nature of data that didn't allow to establish a temporal timing between the events, the evaluation, in Group 1, to be merely subjective, being the analyzed data taking into account the complaints described by the patients and the difficulty in quantifying the insomnia for being a symptom that is sometimes hard to isolate from OSA. It is also important to refer that, in Group 2, for the values of AHI to be more precise, it should be confirmed by the polissonography that consists the "gold standard" to diagnose the sleep pathology. The portable device, such as Stardust ${ }^{\circledR}$, aren't so reliable, due to many factors: the technician being absent during the exam, not allowing the classification of the sleep stages, not being able to evaluate the repercussions of the respiratory disorders in the sleep structure and AHI value may, in many situations, not correspond to reality, since its calculated according to the total time of register, and not according to the time that the patient actually sleeps. The presence of AHI inferior to 5 may not exclude the existence of OSA..$^{7-9}$ Therefore, Drummond et al. ${ }^{37}$ state some studies that demonstrate that the detention of respiratory events with domiciliary studies was identical to the ones found in studies carried out in laboratory, verifying a sensibility and specification of the first of 95 and $96 \%$, respectively.

\section{Conclusion}

In this study the main conclusion was the verification of an association between peri and post menopause and sleep disorder. This association was patented by the high percentage of women in peri or post menopause with evidence of OSA and by the deterioration of symptomatology regarding bad sleep quality after entering peri or post menopause. In the sample studied, the predominance of women with BMI superior to $25 \mathrm{~kg} / \mathrm{m}^{2}$, also suggests that peri and/ or post menopause can effectively be associated to a larger tendency for weight gain. However, it should be necessary more studies in this area, to identify the mechanisms and to understand the complex relationship between the sleep and the hormonal function, investing in the premature prevention and intervention as efficient ways to reduce prevalence of these disorders in peri and post menopause.

It was verified, that within women in Group 2 that took medication to sleep, $80 \%$ had evidence of OSA, which can lead to a new question: Is it the medication to sleep that increases the predisposition for OSA? Furthermore, it was also verified that all women from this Group snored, the roncopathy index, minimum and maximum, registered was of $1.2 \mathrm{events} /$ hour and 53.4 events/hour, respectively. Another question can be made: Can menopause also influence the increase 
of roncopathy index? Curiously, many studies also suggests new researches to determine the increase of prevalence of snoring and/ or OSA, it may be due to other factors related with age/aging (more specifically the changes of neck muscle tones) and not due to the hormonal changes that occur in menopause. . $, 5,25,26^{2}$

Other studies in this are that can also be interesting are: investigate more deeply what is the influence of hormonal substitution therapy used (with estrogens or with estrogens and progesterone) in sleep disorder, since the conclusions of many studies are contradictory according to its benefit, test if menopause has influence in the increase of prevalence of RERA-Respiratory Event Related Arousals and determine if prevalence of member periodic movements in women increase after entering peri or post menopause because these movements can also be responsible for many complaints of insomnia narrated by these women. ${ }^{38}$

To finish, it is important to refer that the evaluation of the sleep disorders should be a priority in women that are in peri and post menopause, mainly when they narrate specific complaints of depression, extreme tiredness, irritability and anxiety that may constitute atypical OSA symptoms. Furthermore, the complaints regarding sleep are most of the times considered, by women in peri and post menopause and by health professionals, as normal, concomitant to vasomotor symptoms and other observed symptoms in these life stages. Thus, sleep respiratory disorders can be frequently devaluated and under diagnosed, aggravating the women's health situation.Therefore, it is imperative to invest in resources and efforts in a larger divulgation of sleep disorder, as well as the peculiar way how it is manifested in women in peri and post menopause.

\section{Acknowledgments}

a) To the Cardiopneumologist Rosa Fonseca for accepting to be my supervisor, for the constant optimism, for the gift of being able to calm me, for all the time spent helping me (it won't be forgotten), for the suggestions and knowledge transmitted, for being a professional example and showing me how to work with competence, dedication and enthusiasm, for all the incentives, for the support, friendship and partnership and for accepting me as I am.

b) To GASIN: Air Products Group for making possible to carry out this project and for all the supplied material.

c) To the health professionals of GASIN: Air Products Group for the essential help in gathering data.

d) To the Teacher Cristina Baeta for the orientation, advice and friendship throughout these long four years.

e) To the Teacher Sandra Silva, for the help and for all the suggestions for the statistic treatment of the gathered data.

f) To all the Teachers of the Cardiopneumology degree who throughout these years have transmitted me all their knowledge to be a good professional and for implementing the constant willing to learn.

g) To all the patients that participated in this study, for the availability and sympathy with which I was received and for understanding the importance of this project.

\section{Conflicts of interest}

Authors declare that there are no conflicts of interest.

\section{References}

1. Gonzalez MPGP, Martinez PAS, Fernandez MTB, et al. Sleep disturbance in menopause. Salud Total de la Mujer 3(3):163-171.

2. Escobar-Cordoba F, Chica-Urzola HL, Cuevas-Cendales F. Sleep disorders related to the female menopause and their treatment. Rev Colomb Obstet Ginecol. 2008;59(2):131-139.

3. Eichling P. Evaluating and treating menopausal sleep problems Menopause Management. 2002;11:8-17.

4. Regal A, Friend CM, Cebrian E. Sleep and women. Revista de Neurologia. 2009;49(7):376-382.

5. De Campos HH, Bittencourt LR, Haidar MA, et al. Sleep disturbance prevalence in postmenopausal women. Rev Bras Ginecol Obstet. 2005;27(12):731-736.

6. Vigeta SMG. Sleep alterations and menopause:a revision of literature. Revista Ciencia, Cuidado e Saude. 2007;6(3):377-383.

7. Couto A, Ferreira JMR. Functional respiratory study-physiological basis and application in clinical practice. Portugal; Lidel Edicoes Tecnicas, 2004

8. Rente P, Pimentel T. The pathology of sleep. Lidel Edicoes Tecnicas, Lisbon, Portugal.

9. Contreras A (2009) Syndrome of obstructive sleep apnea:diagnosis and treatment. Rev Med Clin Conde. 2004;20(4):458-469.

10. Silva GA, Giacon LAT. Sindrome das apneias/hipopneias obstrutivas do sono (SAHOS). Medicina Ribeirao Preto. 2006;39(2):185-194.

11. Fernades MJA. Obstructive sleep apnea syndrome and obesity. Revista da SPCNA. 2006;12(2):41-57.

12. Viana M, Mesquita J, Azevedo P, et al. Indice de massa corporal e sindrome de apneia obstrutiva do sono:Estudo retrospectivo. Revista Portuguesa de Endocrinologia, Diabetes e Metabolismo. 2008;3(2):913

13. Boleo-Tome J, Salgado S, Oliveira AS, et al. Prevalence of cardiovascular disease in a population of patients with obstructive sleep apnea syndrome. Revista Portuguesa de Clinica Geral. 2008;24(3):355-361

14. Daltro CHC, Fontes FHO, Santos-Jesus R, et al. Obstructive sleep apnea and hypopnea syndrome (OSAHS):association with obesity, gender and age. Arq Bras Endocrinol Metab. 2006;50(1):74-81.

15. Schwartz AR, Patil SP, Laffan AM, et al. Obesity and obstructive sleep apnea:pathogenic mechanisms and therapeutic approches. Proc Am Thorac Soc. 2008;5(2):185-192.

16. Andrea Barral Martins AB, Tufik S, et al. Physiopathology of obstructive sleep apnea-hypopnea syndrome. Journal Brasileiro de Pneumologia. 2007;33(1):93-100.

17. Dancey DR, Hanly PJ, Soong C, et al. Impact of menopause on the prevalence and severity of sleep apnea. Chest. 2001;120(1):151-155.

18. Souza CL, Aldrighi JM, Filho GL. Quality of sleep of climacteric women in Sao Paulo:some significant aspects. Rev Assoc Med Bras. 2005;51(3):170-176.

19. Osio G. Trastornos del sueño durante la menopausia. Revista Colombiana de Menopausia 2001;7(1):21-68.

20. Antunes S, Marcelino O, Aguiar T. Pathophysiology of menopause. Revista Portuguesa de Clinica Geral. 2003;19(4):353-357.

21. Ros D, Castell JA, Gascón FS, et al. Menopausia y sueno. Revista Electronica Semestral de Enfermeria. 2004;1(4):17.

22. Bocchino S. Aspectos psiconeuroendocrinos de la perimenopausia, menopausia y climaterio. Rev Psiquiatr Urug. 2005;70(1):66-79. 
23. Ramos MF, Uribe MO. Gonadal hormones and perimenopausal depression. Rev Colomb Psiquiatr. 2008;37(2):236-246.

24. Soares CN. Insomnia during menopause and perimenopauseclinical characteristics and therapeutic options. Rev Psiquiatr Clin. 2006;33(2):103-109.

25. Bixler EO, Vgontzas AN, Lin HM, et al. Prevalence of sleep-disordered breathing in women:effects of gender. Am J Respir Crit Care Med. 2001;163(3 Pt 1):608-613.

26. Shahar E, Redline S, Young T, et al. Hormone replacement therapy and sleep-disordered breathing. Am $J$ Respir Crit Care Med. 2003;67(9):1186-1192.

27. Young T, Finn L, Austin D, et al. Menopausal status and sleep-disordered breathing in the Wisconsin Sleep Cohort Study. Am J Respir Crit Care Med. 2003;167(9):1181-1185.

28. D'Ambrosio C, Stachenfeld NS, Pisani M, et al. Sleep, breathing, and menopause:the effect of fluctuating estrogen and progesterone on sleep and breathing in women. Gend Med. 2005;2(4):238-245.

29. Montplaisir J, Lorrain J, Denesle R, et al. Sleep in menopause:differential effects of two forms of hormone replacement therapy. Menopause. 2001;8(1):10-16.

30. Anttalainen U, Saaresranta T, Aittokallio J, et al. Impact of menopause on the manifestation and severity of sleep-disordered breathing. Acta Obstet Gynecol Scand. 2006;85(11):1381-1388.
31. Franco $\mathrm{C}$, et al. Sindrome da apneia-hipopneia obstrutiva do sono:avaliacao clinica e polissonografica. Neurobiologia. 2009;71(4):1930 .

32. World Health Organization. Obesity: preventing and managing the global epidemic. World Health Organization, Geneva. 2000.

33. Iber C, Ancoli-Israel S, Chesson AL, et al. The AASM manual for the scoring of sleep and associated events: rules, terminology, and technical specifications. Westchester, USA: American Academy of Sleep Medicine; 2007

34. Shin C, Lee S, Lee T, et al. Prevalence of insomnia and its relationship to menopausal status in middle-aged Korean women. Psychiatry Clin Neurosci. 2005;59(4):395-402.

35. Young T. Menopause, hormone replacement therapy, and sleepdisordered breathing:are we ready to heat? Am J Respir Crit Care Med. 2005;163(3 Pt 1):597-601.

36. Rosenthal LD, Dolan DC.The epworth sleepiness scale in the identification of obstructive sleep apnea. J Nerv Ment D. 2008;196(5):429-431.

37. Drummond M, Winck JC, Pereira S, et al. Sindroma de apneia obstrutiva do sono e doença cardiovascular:estudo retrospectivo. Rev Port Pneumol. 2003;9(4):327-335.

38. Pestana H, Gageiro J. Analise de dados para ciencias sociais: a complementaridade do SPSS. 4th edtion. Portugal: Edicoes Silabo; 2005. 